\title{
The three different phases in the dynamics of chemical reaction networks and their relationship to cancer
}

\author{
David B. Saakian ${ }^{1,2}$ and Laurent Schwartz ${ }^{3, \text { * }}$ \\ ${ }^{1}$ Yerevan Physics Institute,2 Alikhanian Brothers St., Yerevan 375036, Armenia \\ ${ }^{2}$ Institute of Physics, Academia Sinica, Nankang, Taipei 11529, Taiwan \\ 3 EcolePolytechnique, Laboratoire d'Informatique, 91128 Palaiseau France and \\ 4 Service d' oncologie, Hopital Raymond Poincare, Garches
}

\begin{abstract}
We investigate the catalytic reactions model used in cell modeling. The reaction kinetic is defined through the energies of different species of molecules following random independent distribution. The related statistical physics model has three phases and these three phases emerged in the dynamics: fast dynamics phase, slow dynamic phase and ultra-slow dynamic phase. The phenomenon we found is a rather general, does not depend on the details of the model. We assume as a hypothesis that the transition between these phases (glassiness degrees) is related to cancer. The imbalance in the rate of processes between key aspects of the cell (gene regulation, protein-protein interaction, metabolical networks) creates a change in the fine tuning between these key aspects, affects the logics of the cell and initiates cancer. It is probable that cancer is a change of phase resulting from increased and deregulated metabolic reactions.
\end{abstract}

PACS numbers: 87.18.-h, 75.10.Nr

\section{INTRODUCTION}

When observing the life on the ground of statistical physics of complex systems we see a hierarchial level of organization and modularity. How can we describe the "good, natural" relations between different parts of complex system? In statistical physics we describe different systems through different order parameter, and in equilibrium different parts of the system have the same temperature. For the stability of the system, it is reasonable to assume the similarity of order parameter at different hierarchy levels and parts (modules) of the system. There is a nice similarity with the no-arbitrage condition in financial markets [1] where different stocks can fluctuate in equilibrium have only identical order parameters, defines as a ratio of driven and diffusion motions. The Random Energy Model (REM) 2] and related complexity (replica symmetry breaking) order parameters [3] work starting from the proteins [4] till quantum chromo-dynamics and strings, therefore we assume that the cell organization and cancer should not be exclusions, there is a possibility that REM related ideas can work there. Using the known results of REM, we will prove that the phase structure of the chemical reaction network kinetics is related with the probabilistic distributions of different chemicals in the steady state distribution. Then we will speculate about the origin of the cancer using similar complexity ideas.

One of the main ideas for cell modeling is to consider the dynamical models with network structure [5-7]. One can realize this program studying the network of catalytic reactions, identifying the phenotype of the cell with the attractor of the nonlinear system of differential equations.

*Electronic address: saakian@yerphi.am
While constructing the kinetic constants, it is advantageous to have a detailed balance condition. The density of different chemical components change according to the kinetic constants, and the latter are defined through the energies of that components. We are following [9]. There are $M$ chemical components. The transformation between chemicals $X_{i}$ and $X_{j}$ is catalyzed by some component $x_{c}$, so has a rate $k_{i, j} x_{c} x_{i}$

Thus we can write a set of equations [9]

$$
\frac{d x_{i}}{d t}=\sum_{j, c} W(i, j, c) x_{c}\left(\left(k_{j, i} x_{i}-k_{i, j} x_{i}\right)\right.
$$

where $W(i, j ; c)=W(j, i ; c)=1$ when there is a reaction and zero otherwise. The kinetic coefficients are defined through the energies of the chemical component $E_{i}$ and some inverse temperature $\beta$ [9] $k_{i, j}=$ $\min \left\{1, \exp \left[\beta\left(E_{i}-E_{j}\right)\right]\right.$. Thus the kinetic coefficients are defined through the energy landscape. The choice of $k_{i, j}$ is quite reasonable for catalytic reaction network: there is some finite rate when the reaction goes to the low energy configuration, while there is a small probability for the reaction in inverse direction.

The Eq.(1) has a steady state solution

$$
x_{i}^{s}=\frac{\exp \left(-\beta E_{i}\right)}{\sum_{j} \exp \left[-\beta E_{j}\right]}
$$

In [9] it was considered a homogenous distribution of energies in some interval $[0, \epsilon]$ and at some temperatures glassy behavior was found. The authors claimed that the same is the situation in case of normal distribution, while the phenomenon lacks in case of log-normal distribution or the distribution with the tail. 


\section{RESULTS}

The statistical physics phases of the model. Consider a general distribution for energy levels

$$
\rho\left(E_{i}\right)=\frac{1}{2 \pi} \int_{-i \infty}^{i \infty} d h \exp [-h E+\ln (M) \phi(h)]
$$

where $\phi(h)$ is a some function. For the normal distribution we have $\phi(\beta)=\beta^{2} / 2$.

When considering pure deterministic dynamics with $M$ degrees, we can map our model to the statistical physics model REM [2] with ln $M$ degrees assuming large $\mathrm{M}$ and independence of energy distribution.

In our model $E_{i}$ are the energies connected with different chemical components of the model.

Nevertheless let us introduce a "partition function" Z, $Z=\sum_{i=1}^{M} x_{i}^{s}$. The statistical physics model with the partition function $\mathrm{Z}$ has two phases. At small $\beta$ it is in the paramagnetic phase $(\mathrm{PM})$ with fast relaxation. When the following equation has a real positive solution $\beta_{c}$ [10]:

$$
\left(1+\phi\left(\beta_{c}\right)\right)-\beta_{c} \phi^{\prime}\left(\beta_{c}\right)
$$

the second, spin-glass phase (SG) is possible in the model at $\beta>\beta_{c}$. Two phases have different probability distributions for the $\mathrm{Z}$ in the statics [1]. While formulated in spin-spin interaction version, in SG phase there is a slow relaxation, see also [12]. Thus we related some statistical physics with the quenched disorder of the dynamic model (1).

How can we distinguish two phases? At different phases there are different distributions functions for Z, and also different expressions for the order parameter $m=1-\frac{\sum_{i}\left(x_{i}^{s}\right)^{2}}{\left(\sum_{i} x_{i}^{s}\right)^{2}}$.

One has two expressions for the order parameter (connected with replica symmetry breaking) [13]

$$
\begin{gathered}
m=1, \beta<\beta_{c}, \\
m=\frac{\beta_{c}}{\beta}, \beta>\beta_{\mathbf{c}}
\end{gathered}
$$

For the normal distribution case $\beta_{c}=\sqrt{2}[2]$. While there are $M$ degrees in our dynamic model instead of $\ln M$ spins in related REM, we observed the phase transition in the dynamics, found first in [9]. A similar model has been solved in [12].

In the PM phase the order parameter $m(t)$ converges to the steady state value as [12]

$$
|m(t)-1| \sim \exp \left[-a_{1} * t\right]
$$

while in the SG phase [12]

$$
|m(t)-m| \sim \exp \left[-a_{2} * t^{m}\right]
$$

where $a_{1}, a_{2}$ are some constants.
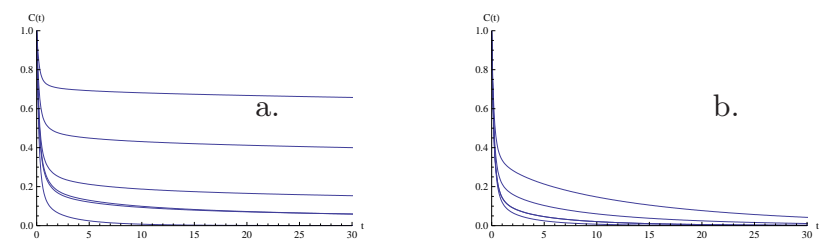

FIG. 1: The $C(t)=\frac{\sum_{i}\left(x_{i}^{c}-x_{i}(0)\right)\left(x_{i}^{c}-x_{i}(0)\right)}{\sum_{i}\left[\left(x_{i}^{c}-x_{i}(0)\right]^{2}\right.}$ vs time. $N=100$. Every chemical is connected with $K=10$ other chemicals.(a) The low line has no ferromagnetic configuration, $\beta=0.8 \beta_{c}$, the next line has $\beta=\beta_{c} * 1.4$, no ferromagnetic configurations. the higher lines correspond to the $\beta=1.4 \beta_{c}$ and ferromagnetic configurations with the relative concentration $0.08,0.15,0.30,0.60$. (b) $\beta=\beta_{c} * 0.8$, there is no ferromagnetic configurations. the higher lines correspond to the $\beta=1.4 \beta_{c}$ and ferromagnetic configurations with the relative concentration $0.08,0.15,0.30,0.60$.

Let us now add a special energy level $E_{0}=-J_{0} \ln M$. When $J_{0}>\beta_{c}$, third, ferromagnetic phase (FM), is possible [3]. We investigated the dynamics of the model in this case. When the steady state concentration of the "ferromagnetic" chemical is comparable with the total concentration of the system, the reaction dynamics is becoming slower than even in the SG phase for the initial homogenous distribution. The reason of ultra-slow relaxation is the energy gap between the ferromagnetic level and other levels. We performed a numeric for the case of normal distribution (realistic for the biology of the cell [15], [16]) with $M=100, K=10$ see Fig. 1 , and observed the phase transition phenomenon in the dynamics. The SG phase Fig 1a is much more sensitive to the appearance of FM configuration, than the PM phase, Fig 1b.

This model can be applied for the reactions in the cell. We assume that the normal chemical kinetics of the cell corresponds to the SG phase, contrary to PM or FM phases.

\section{How general are these results?}

To obtain two phases we assume that: a. The nonlinear system of equation has one steady state solution and b. randomness of steady state concentrations $x_{i}^{s}$. The second condition is well established experimentally for the concentration of chemicals in the cell 16]. The concentration of chemicals fluctuate from cell to cell. We used a concrete form of the nonlinear dynamic. Let us consider another version of nonlinear dynamics, i.e. $\frac{d x_{i}}{d t}=\sum_{j, c} W(i, j, c)\left(x_{c}\right)^{2}\left(k_{j, i} x_{i}-k_{i, j} x_{i}\right)$. We performed numerics and found the same qualitative picture: the statistical physics phase for the quenched disorder defines the phase of the dynamic. If we consider the relaxation from the initial configuration near the steady state, then the results of different nonlinear attractor models certainly are similar; they are defined mainly by $k(i, j)$.

How can we apply these results to the cell-cell interaction, gene expression problem? Ao formulated well the idea of adaptive landscape for bio-networks [8]. In the 
Ao's approach, while there is a noise, transversal (magnetic like) field and friction, the system has a given steady state defined by a potential landscape. This potential landscape should be identified with the fitness. The latter sometimes is directly connected with the ordinary free energy. This idea have been applied to the cancer analysis [17]. The robustness and plasticity aspects in the dynamics of the cancer cells have been considered in [18] and [19]. The key point here is again the concept of the landscape again. Again fast and slow phases in the dynamics are possible, for our derivation was important just the steady state distribution of energy (fitness). We considered SG-PM phase transition at different temperatures. The same transition happens when the steady state distributions are changed. This is an important observation as the temperature is rather a constant in biological systems.

Connection between the phase in cell's chemical kinetics and the type of fitness landscape.

According to our classification, the steady state could be identified either with SG, FM, or PM ones. The other characteristic of the model are the basin of attractions of these steady states. It is an advantage to have large basins of attractions, but simultaneously a slow relaxation dynamics.

A very interesting point is the connection of behavior of the cell (basin of attraction + phase of quenched disorder) with the evolution behavior. Here it was found that the dynamics of the evolving population drastically depends on the character of the fitness landscape function: the FM, or SG or their border case, FM-SG [20]. It is highly intriguing if the character of fitness function of evolving cell will be the same as the character of the chemical reaction kinetics. A similar ideas about the tight relation between cell processes and evolution characteristics has been suggested in a series of articles by K. Kaneko and co-workers, [18], where they claim that the large basin of attraction, related with the given phenotype, can be connected with the mutational robustness.

In [3] also speculations are made about resonance in complex systems, assuming identical complexity parameters. It has been assumed that the possibility of parametric amplification of the motion (resonance) and parametric fast attenuation (anti-resonance) is one of the key features of normal living systems. Such a property can exist for some parameters of dynamic system, without being present for other values.

\section{Speculations about cancer.}

How can we understand the cancer? For a recent review see [21]. Here we distinguish two aspects: the origin and the dynamic behavior. The evolution dynamics aspect of cancer is well know since [22]-24]. The key role of metabolism is also well recognized 25]. Looking for simple and general origin for the cancer, we assume the following hypothesis. Since the start of the life, there is some fine tuning between different key aspects of the life such as gene regulation, metabolism, $\mathrm{pH}$, cytoskeleton. It appears that these different aspects of life are inter- twined, and their interaction defines the decision making, the logics of the cell [26].

The fine tuning of the cell should be understood as an existence of proper statistical physics phases, identical complexity parameters, oscillations, and this fine tuning is supposed to be increasing in parallel with the evolution. According to our hypothesis, in the case of cancer: external aggression like chronic inflammation or increased cellular metabolism caused by oncogene activation, disrupts the degree of the fine tuning, change the reaction rates and as a result, the decision making, the logics of the cell.

Having less degree of fine tuning, the cell, in some sense, returned back in evolution history, using old machinery. A candidate of such a poor fine tuning, can be the change in the reaction rates (glassiness), discussed in the previous section. The slow, glassy like dynamics is typical for the cell to support the non-equilibrium in the cell, an important aspect of the life [27]. Another side of phenomenon is connected with the memory processes, the glassy (slow) phase possess such memory, while the fast phase does not. The necessity of glassy dynamics is well recognized in case of immune system 28]. We assume that the cancer cell has another version of glassiness than the healthy cells. It is intriguing that the osmotic pressure plays a crucial role for both cancer [29] and cytoskeleton glassiness [27]. In case of metabolic network the cancer cells choose a fast dynamics, and perhaps the reaction network system is in the PM phase.

The fast reaction rate and less fine tuning are tightly related. Normal cells can either be in anabolic or catabolic state. They use oxygen and burn, for example, glucose. It results energy, water and Carbonic gas. The alternative pathway is anabolism. They use energy water and Carbonic gas to synthesize for example glucose. The cells can synthesize other compounds like DNA, RNA, cholesterol.... In normal cells the two pathways cannot be done at the same time. But at different time of the day one cell can either be in anabolic or in catabolic phase. In cancer cells, some enzymes (modules) are on the anabolic mode, some others are on the catabolic mode. It results a deregulation of the metabolism which could be seen as a different phase 29].

Consider the change of decision making mechanisms. Probably this is the start point of cancer. The metabolic rate and decision making are key features of living matter [30], 31]. They should be considered both on a single cell level, and in a cell-cell ( tissular) interaction [32][33], [34]. We should carefully analyze the metabolic network [35] and decision making in the healthy cell, and compare them with those in the cancer cell. According to [25], the cancer cell is less dependent on the surrounding constraints than the healthy cell. An intriguing possibility is the de-synchronization in decision making between healthy cells and cancer cells, for example due to lack of polarity in cancer cells 36]. Fortunately the concept of de-synchronization is already generalized for 
non-harmonic processes [37.

We mentioned already the hypothesis of [3] about a property of living system to reveal both resonance and anti-resonance features. In case of cancer the second property (apostasis) is lost. In [6] it was suggested to construct a rather simplified models of cells so as to capture key features of the cancer phenomenon, instead of looking into too complicated models. In case we try to give a simplified phenomenological model of cancer via some dynamical model, we should take care about the mentioned feature of the model (a presence of resonance and anti-resonance).

\section{DISCUSSION}

We checked the existence of different phases (degree of glassiness) in the chemical reaction network dynamics: fast, slow and ultra-slow, connected with the quenched disorder. While the first two phases has been found numerically in [9], we determined the conditions when they emerge and also found the third phase. The phenomenon exists only for the special distributions of chemicals with a nontrivial solution for Eq.(6), which related to the distribution of proteins in the cells [25]. Looking for a simple and complexity related key reason for the cancer, we suggested a hypothesis that the healthy and cancer cells have different phases in the chemical reaction network dynamics, connected with the glassy properties. The glassy like properties of statistical physics models are not artifacts of the modeling, they have very deep and universal meaning as complexity order parameters. One can apply the spin-glass order parameters, statistical physics phases, to the immune system [28], proteins [4], cytoskeleton [27], the reaction network (the current work result). In the cells, both normal and malignant, there are many modules playing the role of logical units. The cancer cell has a poor fine tuning of different modules, some of these modules change their meaning, affecting the decision making aspects of the cell. We should perform a careful analysis of different aspects in metabolic reactions [38], gene regulatory network, protein-protein interaction network, cytoskeleton dynamics to understand different choices in case of the healthy cell and cancer, the difference in a decision making schemes. We should verify our hypothesis, that different aspects of the cell (organism) should share the same complexity order parameters. We emphasize the idea of "energy" landscape in modern modeling of cell dynamics, including population dynamics. We found that the statistics of distribution of different degrees at peaks of the landscape is as important as the landscape itself. The landscape models like the ones [17, [8] should be completed also by a description of statistics of different components at peaks. We suggest to try experimentally measure both the distribution of different chemicals in healthy and cancer cell, and also compare the rate of (metabolic) reactions, trying in this way derive quantitative criteria to identify the transition between SG (healthy, slow) and PM (fast) phases. Besides identifying different statistical physics phases of chemical reaction networks, we suggest to focus on decision making synchronization by cells and the existence of anti-resonance property.

It is possible that the investigation of the advanced evolution models, incorporating some decision making aspects, will improve our understanding of both the cancer and the origin of life. Only genetical aspects or metabolism are not sufficient to describe the origin of life [39].

DBS thank the DARPA prophecy project, NSC 1012923-M-001-003-MY3 and NCTS (North) in Taiwan, for the support, K. Kaneko, H. Q. Chen, T. J. Kobayashi for discussions.

\section{References}

[1] E. Derman, Quantitative Finance 2 (2002) 282.

[2] B. Derrida, Phys. Rev. B. 24 (1981) 2613.

[3] D.B. Saakian, Phys. Rev. E, 71 (2005) 016126.

[4] V. S. Pande, A. Y. Grosberg and T. Tanaka, Rev. Mod. Phys. 72 (2000) 259.

[5] S. Kauffman, J. Theor. Biol. 22 (1969) 437.

[6] K. Kaneko Life: An Introduction to Complex Systems Biology, (Springer), 2006.

[7] L. Kadanoff, Physics today, 62 (2009) 8.

[8] P. Ao, 36, (2009) 63.

[9] A. Awazu, K. Kaneko, Phys. Rev. E, 80(2009) 041931.

[10] D. B. Saakian, Phys. Rev. E. 85 (2012) 011109.

[11] E. Gardner, B. Derrida, J. Phys. A22 (1989) 1975.

[12] C. De Dominicis, H. Orland, F. Lainee, Journal De Physique Letters, 46 (1985) L463.

[13] D.J. Gross, M. Mezard, Nucl. Phys. B 240 (1984) 431.
[14] K. Kaneko,C. Furusawa,PRL 108 (2012) 208103.

[15] C. Furusawa, T. Suzuki, A. Kashiwagi, T. Yomo, K Kaneko, Biophysics 1, (2005) 25.

[16] H. Salman, N. Brenner, C.-K. Tung, N. Elyahu, E. Stolovicki, L. Moore, A. Libchaber,and E. Braun, PRL 108 (2012) 238105.

[17] P. Ao, D. Galas, L. Hood, X. Zhu, Medical Hypotheses, 70, (2008) 678.

[18] K. Kaneko, Bioessays, 33 (2009) 403.

[19] R. A. Gatenby, R. J. Gillies, J. S. Brown, Nature reviews Cancer, 10 (2010) 526.

[20] D. B. Saakian and J. F. Fontanari, Phys. Rev. E 80 (2009) 041903.

[21] D. Hanahan, R. A. Weinberg, Cell, 144 (2011)646.

[22] P. C. Nowell, Science 194 (1976) 23.

[23] J. Erenpreisa and M. S. Cragg,PP. 61, in: P. Pontarotti 
(Editor), Evolutionary Biology from Concept to Application, (Springer-Verlag, Berlin), 2008.

[24] M. Graves, C.C. Maley, Nature, Nature486 (2012) 306.

[25] M. A. Lazar and M. J. Birnbaum,Science 336 (2012) 1651.

[26] P. Nurse, Nature, 454 (2008) 424.

[27] P. Bursac, G. Lenormand, B. Fabry, M. Oliver, D. A. Weitz, V. Viasno, J. P. Butler, and J. J. Fredberg. Nature Mater, 4, (2005) 557.

[28] J. Sun, D. J. Earl, and M. W. Deem, Phys. Rev. Lett. 95 (2005) 148104.

[29] M. Israel and L. Schwartz, The metabolic advantage of cancer cells Molecular Cancer, 10(2011) 70.

[30] L.H. Hartwell, J.J. Hopifield, S. Seibler, A.W. Murray, Nature, 402 (1999) C47.

[31] N. Murphy, G. F. R. Ellis, and T. O'Connor (Eds.),
Downward Causation and the Neurobiology of Free Will, (Springer-Verlag Berlin Heidelberg) 2009.

[32] T. Hellikar, J. Konvalina, J. Heidel, J.A. Rogers, PNAS, 105 (2008) 1913.

[33] T. J. Kobayashi, PRL 104,(2010) 228104.

[34] M. D. Brennan, R. Cheong, A. Levchenko, Science 338 (2012) 334 .

[35] J. Loscalzo, A. L. Barabasi, Systems biology and the medicine, 3 (2011) 619.

[36] S. Ohsawa, K. Sugimura, K. Takino, T. Xu, A. Miyawaki, T. Igaki, Dev. Cell. 202011315.

[37] S. Acharyya and R. E. Amritkar, EPL, 99 (2012) 40005.

[38] O. Warburg, Science, 123 (1956) 309.

[39] V. Vasasa, E. Szathmary, and M. Santosa, PNAS 107 (2010) 1470. 